\title{
Anisotropy Distribution and Magnetoimpedance in Stress Annealed Amorphous Ribbons
} J.L. Muñoz ${ }^{1}$, G.V. Kurlyandskayaa ${ }^{1,2}$, J.M. Barandiarán ${ }^{1}$, A.P. Potapov ${ }^{2}$, M. Vázquez ${ }^{3}$

'Dpto. Electricidad y Electrónica, Universidad del País Vasco UPV-EHU, P.O. Box 644, 48080 Bilbao, Spain

${ }^{2}$ Institute of Metal Physics UD RAS Kovalevskaya str. 18, 620219 Ekaterinburg, Russia ${ }^{3}$ Instituto de Magnetismo Aplicado UCM-RENFE and Instituto de Ciencia de Materiales de Madrid, CSIC, P.O. Box 155, 28230 Las Rozas (Madrid), Spain

Kevwords: Amorphous ribbons, magnetoimpedance, induced anisotropy, domain structure.

\begin{abstract}
The magnetic anisotropy and the magneto-impedance of stress annealed $\mathrm{FeCoCrSiB}$ amorphous ribbons have been studied. Selected heat treatments have been choosen in order to induce, at different conditions, the same value of transverse induced anisotropy constant. These samples, showing nearly identical longitudinal hysteresis loops, exhibit different MI maximum value, MI curve shape and MI hysteresis. These results can be explained assuming a distribution of induced magnetic anisotropy constants, whose parameters depend on the heat treatment conditions. It is shown that even in the absence of the anisotropy distribution, theoretical magnetoimpedance ratio never reaches an infinite value when applied field equals the anisotropy field of the sample.
\end{abstract}

\section{Introduction}

Magnetoimpedance (MI) effect in amorphous wires and ribbons has been extensively studied during the last years $[1,2]$ in an effort to use it as the basis for tiny and fast field sensors and for magnetic recording heads. MI consists of the change of the impedance felt by an AC current that flows through a magnetic material when an external DC field is applied. This effect is a consequence of the change of transverse permeability of the sample with the biasing field [3], so there is a direct relationship between magnetic characteristics such as domain structure or induced anisotropies with MI behavior. Recently, Co-rich stress-annealed ribbons, with a transverse domain structure, have been used to study this relationship [4,5]. This is because the evolution of the transverse permeability with the bias field can be more easily visualized than in other samples with a more complicated domain structure. However, the resulting magnetic domain structure of these samples can be very complex [6] and the induced anisotropy constant value is not the only parameter determining the MI behavior. In this work, we compare the behavior of two samples that, having the same value of induced magnetic anisotropy constant, exhibit different MI characteristics.

\section{Experimental results}

Amorphous ribbons with composition $\mathrm{Fe}_{3} \mathrm{Co}_{67} \mathrm{Cr}_{3} \mathrm{Si}_{15} \mathrm{~B}_{12}$ were obtained by melt spinning. They were $22 \mu \mathrm{m}$ thick, $1.1 \mathrm{~mm}$ wide and $100 \mathrm{~mm}$ long. Their Curie and crystallization temperatures were 160 and $570{ }^{\circ} \mathrm{C}$ respectively. Each sample was pre-annealed in vacuum at $\mathrm{T}=350$ ${ }^{\circ} \mathrm{C}$ for 1 hour. After such pre-treatment the samples were stress annealed under several conditions: $\mathrm{T}=250^{\circ} \mathrm{C}, \sigma=1320 \mathrm{MPa}$ for 4 hours (sample S1) and $\mathrm{T}=350^{\circ} \mathrm{C}, \sigma=330 \mathrm{MPa}$ for 1 hour (sample S2). These heat treatments induce a transverse anisotropy in the ribbons, whose magnitude is $\mathrm{K}_{\mathrm{i}}=90 \mathrm{~J} / \mathrm{m}^{3}$ for both samples, as estimated from longitudinal magnetization curves measured using a conventional technique (Fig. 1a). Magneto-optical Kerr effect observations reveal the presence of transverse, equally spaced domains, separated by zig-zag walls [7]. Magnetoimpedance ratio, defined as $\Delta \mathrm{Z} / \mathrm{Z}=\left[\mathrm{Z}(\mathrm{H})-\mathrm{Z}\left(\mathrm{H}_{\max }\right)\right] \times 100 / \mathrm{Z}\left(\mathrm{H}_{\max }\right)$, was measured using a fully computer controlled 
system [7] at a driving current of $\mathrm{I}_{\mathrm{rms}}=3 \mathrm{~mA}$ at a frequency of $2 \mathrm{MHz}$. The results are presented in Fig. 1b. We observe that for both samples a MI maximum is observed at a field close to the anisotropy field as evaluated from the magnetization curves. The maximum for the sample $\mathbf{S} 2$ is higher than that for sample S1. Also, sample S1 presents a much more pronounced magnetoimpedance hysteresis than sample $\mathbf{S 2}$, and the shape of the magneto-impedance peaks is different.
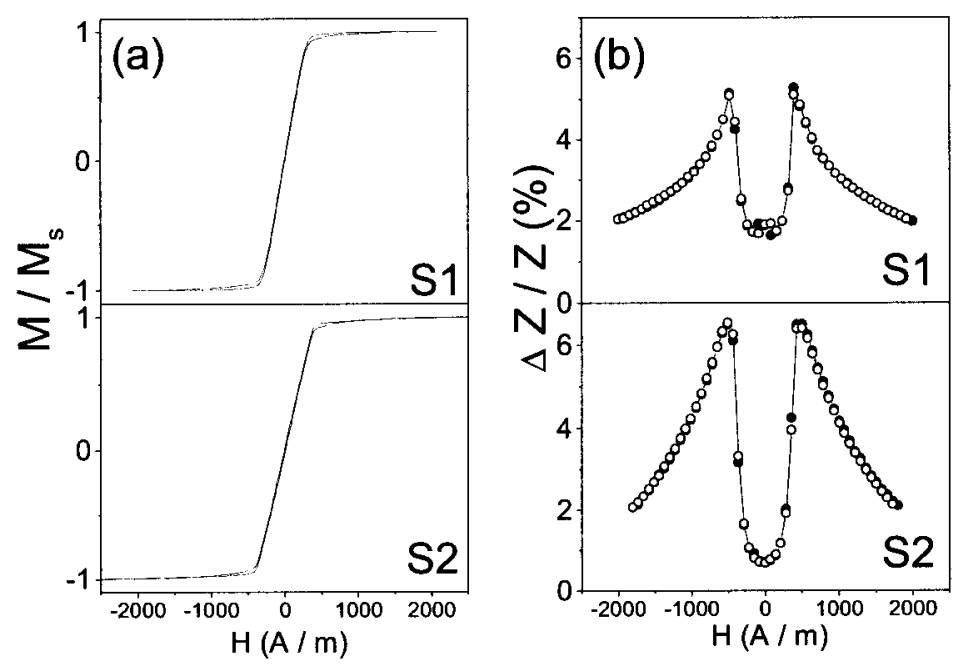

Fig. 1. The hysteresis loops (a) and the magneto-impedance (b) for samples S1 and S2. The samples with the same value of induced anisotropy $K_{i}=90 J / \mathrm{m}^{3}$ show different MI maximum value and MI hysteresis.

\section{Model, numerical results and discussion}

For a ribbon having a transverse domain structure, magnetoimpedance can be understood to be a consequence of the change of transverse permeability $\left(\mu_{D C}\right)$ with the applied DC field $[3,8]$. The actual $\mu_{\mathrm{DC}}$ value depends on the value of the transverse induced anisotropy $\left(\mathrm{K}_{\mathrm{i}}\right)$. In equilibrium at zero applied field the magnetization will lie along the transverse easy direction of the ribbon. The application of the bias field $\mathrm{H}_{\mathrm{dc}}$ in the ribbon direction rotates magnetization vector towards the ribbon axis. If the anisotropy character is pure uniaxial with a single $\mathrm{K}_{\mathrm{i}}$ constant, when $\mathrm{H}_{\mathrm{dc}}$ equals the anisotropy field $\left(\mathrm{H}_{\mathrm{k}}\right)$ the applied field compensates the anisotropy of the sample and then the transverse permeability would become ideally infinite. Actually, $\mu_{\mathrm{DC}}$ never reaches an infinite value even in the ideal case of having a single $\mathrm{K}_{i}$, due to the non-uniformity of the AC field created by the driving current, that spreads out the permeability values. So, a maximum in $\mu_{D C}$, and thus in impedance, is expectable at $\mathrm{H}_{\mathrm{dc}}=\mathrm{H}_{\mathrm{k}}[3]$.

If we consider a reasonable (i.e., gaussian) distribution of $\mathrm{K}_{\mathrm{i}}$ values, centered at the mean value obtained experimentally from the magnetization curves, it is possible to numerically calculate an average transverse permeability. For each anisotropy constant value, and for each $H_{d c}$ value, magnetization will lie in equilibrium on a direction forming an angle $\theta$ with the easy axis. If a DC current is flowing through the sample in the ribbon direction, there will be an additional field acting on $\mathrm{M}_{s}$, that will rotate it out of its equilibrium direction. Thus, we can calculate an average transverse $\mu_{\mathrm{DC}}$ obtaining the change in the transverse component of magnetization, and considering 
the non-uniformity of the internal field. This field will change linearly from 0 at the middle of the ribbon to $\mathrm{H}_{0}=\mathrm{I} / 2 \mathrm{a}$ at the surface, where $2 \mathrm{a}$ is sample thickness and $\mathrm{I}$ is the amplitude of the current.

From the magnetization curves in Fig. 1, we get that the induced anisotropy constant is $\mathrm{K}_{\mathrm{i}}=$ $90 \mathrm{~J} / \mathrm{m}^{3}$. Using this value we can artificially build up several gaussian distributions. Numerical computation of $\mu_{\mathrm{DC}}$ as a function of $\mathrm{H}_{\mathrm{dc}}$ using the magnetic characteristics of the samples and the previously calculated distribution, yield to the permeability curves presented in Fig. 2. In this figure we have also drawn the curve for the case with a single anisotropy constant value (no distribution). The three distributions choosed were 6,18 and $24 \mathrm{~J} / \mathrm{m}^{3}$ wide. As clearly seen, even in the absence of a distribution of $K_{u}$ values, permeability remains always finite. Since the non-uniform field and different distributions result in different transverse permeability curves: the broader the distribution, the smaller the maximum. So, the maximum magneto-impedance ratio will be obtained when there is no distribution of $\mathrm{K}_{\mathrm{i}}$ values.

The anisotropy in these samples is very well controlled by the conditions of the heat treatments. Other studies in samples of similar composition $[6,9,10]$ have shown that the induced anisotropy is a negative uniaxial anisotropy with an easy plane perpendicular to the stress application direction. It increases with increasing annealing temperature. The out-of-plane

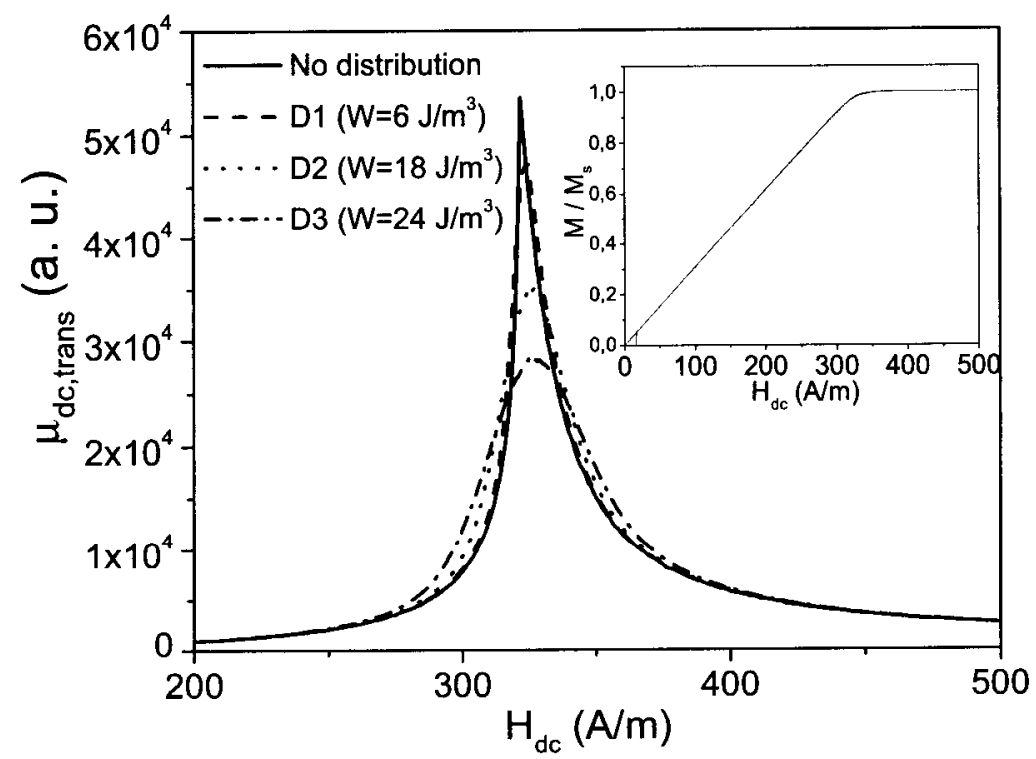

Fig. 2. Calculated transverse permeability for a ribbon with a transverse domain structure and a distribution of anisotropy constant value. $\mathrm{W}$ is the maximum width of the gaussian distribution. The small picture shows the calculated longitudinal magnetization curve for all the cases.

component $\left(\mathrm{K}_{\mathrm{y}}\right)$ of the induced anisotropy in these samples is slightly higher than the in-plane $\left(\mathrm{K}_{\mathrm{z}}\right)$ component. Actually the free energy density $F$ should be written using three anisotropy constants $\mathrm{F}=\mathrm{F}\left(\mathrm{K}_{1 \mathrm{z}}, \mathrm{K}_{1 \mathrm{y}}, \mathrm{K}_{2 \mathrm{y}}\right)$ to describe properly this complicate high-order anisotropy. Fixing $\mathrm{K}_{1 \mathrm{z}}$ stressinduced magnetic anisotropy constant (direction in plane of the ribbon parallel to the applied stress) like $\mathrm{K}_{\mathrm{i}}=90 \mathrm{~J} / \mathrm{m}^{3}$ we may anyway have the uncertainty in the values of the $\mathrm{K}_{1 \mathrm{y}} \mathrm{K}_{2 \mathrm{y}}$ constants depending on the heat treatment. Comparing the results for our two samples, we can say that for sample $\mathbf{S} 2$ the higher annealing temperature leads to a narrower distribution. 
So, in order to obtain a high magnetoimpedance ratio in stress-annealed Co-rich amorphous ribbons, the aim must be to obtain a narrow anisotropy distribution. The maximum MI ratio will be obtained for a perfectly uniaxial transverse anisotropy.

\section{Conclusions}

The MI effect in stress-annealed ribbons has been investigated. Different treatments leading to the same value of induced anisotropy can give rise to different MI curves. We have explained these results assuming that there exists an anisotropy constant distribution. This distribution, although leading to nearly identical magnetization curves, produce quite different MI curves. In addition, it is shown that even in the absence of this distribution, theoretical MI ratio will never get an infinite value when applied field equals the anisotropy field of the sample. Finally, it is concluded that the maximum MI ratio will be obtained when the induced anisotropy is close to perfect uniaxial anisotropy.

\section{Acknowledgments}

Galina Kurlyandskaya would like to thank The Basque Government for the financial support of the Visiting Scientist Grant. We thank Dr. V. A. Lukshina for her help.

\section{References}

[1] R. S. Beach and A.E. Berkowitz., J. Appl. Phys. 76 (1994) 6209-6213

[2] R.L. Sommer and C.L. Chien J. Appl. Phys. 79 (1996) 5139-5141

[3] D.-X. Chen, J.L. Muñoz, A. Hernando and M. Vázquez, Phys. Rev. B, VOL 57, Number 17, May 1998, pp. 10699

[4] G.V. Kurlyandskaya, M. Vázquez, J.L. Muñoz, D. García and J. McCord, J. Magn. Magn. Mat. 196-197 (1999) 259-261.

[5] M. Vázquez, G.V. Kurlyandskaya, J.L. Muñoz, A. Hernando, N.V. Dmitrieva, V.A. Lukshina and A.P. Potapov, J. Phys. IV France 8 (1998), Pr2-143.

[6] A.Hubert and R.Schäfer, Magnetic domains,Springer-Verlag, 1998, p.439.

[7] Kurlyandskaya G.V., Vázquez M., Muñoz J.L., García D., and McCord J.// J. Magn. Magn. Mater., 196-197 (1999), p.259.

[8] Chen D.-X. and Muñoz J.L., IEEE Trans. on Magn. VOL 35, Num. 3, May 1999, p. 1906-1924.

[9] G.V. Kurlyandskaya, N.V. Dmitrieva, A.P. Potapov, V.A. Lukshina, L.M. Voronova, I.V. Gervas'eva and N.G. Bebenin, Phys. Met. Metal. 83 N5 (1997) 41-46.

[10] L. Krauss, N. Zarubova, K. Zaveta and P. Duhaj, Magnetic properties of amorphous metals, Edited by A. Hernando, North Holland, Amsterdam, 1987. 